\title{
Dynamic temporal signal processing in the inferior colliculus of echolocating bats
}

\author{
Philip H.-S. Jen ${ }^{1}{ }^{*}$, Chung Hsin $W_{u^{2}}$ and Xin Wang ${ }^{3}$ \\ ${ }^{1}$ Division of Biological Sciences and Interdisciplinary Neurobiology Program, University of Missouri, Columbia, MO, USA \\ 2 Department of Life Science, National Taiwan Normal University, Taipei, Taiwan, ROC \\ ${ }^{3}$ College of Life Science, Central China Normal University, Wuhan, Hubei, PRC
}

\section{Edited by:}

Manuel S. Malmierca, University of Salamanca, Spain

Reviewed by:

Zoltan M. Fuzessery, University of Wyoming, USA

Donal G. Sinex, Utah State

University, USA

\section{*Correspondence:}

Philip H.-S. Jen, Division of

Biological Sciences, University of

Missouri, Columbia, MO, USA.

e-mail: jenp@missouri.edu
In nature, communication sounds among animal species including humans are typical complex sounds that occur in sequence and vary with time in several parameters including amplitude, frequency, duration as well as separation, and order of individual sounds. Among these multiple parameters, sound duration is a simple but important one that contributes to the distinct spectral and temporal attributes of individual biological sounds. Likewise, the separation of individual sounds is an important temporal attribute that determines an animal's ability in distinguishing individual sounds. Whereas duration selectivity of auditory neurons underlies an animal's ability in recognition of sound duration, the recovery cycle of auditory neurons determines a neuron's ability in responding to closely spaced sound pulses and therefore, it underlies the animal's ability in analyzing the order of individual sounds. Since the multiple parameters of naturally occurring communication sounds vary with time, the analysis of a specific sound parameter by an animal would be inevitably affected by other co-varying sound parameters. This is particularly obvious in insectivorous bats, which rely on analysis of returning echoes for prey capture when they systematically vary the multiple pulse parameters throughout a target approach sequence. In this review article, we present our studies of dynamic variation of duration selectivity and recovery cycle of neurons in the central nucleus of the inferior colliculus of the frequency-modulated bats to highlight the dynamic temporal signal processing of central auditory neurons. These studies use single pulses and three biologically relevant pulse-echo (P-E) pairs with varied duration, gap, and amplitude difference similar to that occurring during search, approach, and terminal phases of hunting by bats. These studies show that most collicular neurons respond maximally to a best tuned sound duration (BD). The sound duration to which these neurons are tuned correspond closely to the behaviorally relevant sounds occurring at different phases of hunting. The duration selectivity of these collicular neurons progressively increases with decrease in the duration of pulse and echo, P-E gap, and P-E amplitude difference. GABAergic inhibition plays an important role in shaping the duration selectivity of these collicular neurons. The duration selectivity of these neurons is systematically organized along the tonotopic axis of the inferior colliculus and is closely correlated with the graded spatial distribution of $\mathrm{GABA}_{A}$ receptors. Duration-selective collicular neurons have a wide range of recovery cycle covering the P-E intervals occurring throughout the entire target approaching sequences. Collicular neurons with low best frequency and short BD recover rapidly when stimulated with P-E pairs with short duration and small P-E amplitude difference, whereas neurons with high best frequency and long BD recover rapidly when stimulated with P-E pairs with long duration and large P-E amplitude difference. This dynamic variation of echo duration selectivity and recovery cycle of collicular neurons may serve as the neural basis underlying successful hunting by bats. Conceivably, high best frequency neurons with long BD would be suitable for echo recognition during search and approach phases of hunting when the returning echoes are high in frequency, large in P-E amplitude difference, long in duration but low in repetition rate. Conversely, low best frequency neurons with shorter BD and sharper duration selectivity would be suitable for echo recognition during the terminal phase of hunting when the highly repetitive echoes are low in frequency, small in P-E amplitude difference, and short in duration. Furthermore, 
the tonotopically organized duration selectivity would make it possible to facilitate the recruitment of different groups of collicular neurons along the tonotopic axis for effective processing of the returning echoes throughout the entire course of hunting.

Keywords: duration selectivity, echolocation, inferior colliculus, pulse-echo pairs, recovery cycle, temporal signal processing

\section{INTRODUCTION}

In nature, communication sounds among animal species including humans are typical complex sounds that vary with time in several parameters including amplitude, frequency, duration as well as separation, and order of individual sounds (Popper and Fay, 1995; Shannon et al., 1995). Among these parameters, sound duration is a simple but important one that contributes to the distinct spectral and temporal attributes of individual biological sounds. Previous studies of selectivity of auditory neurons to sound duration show that most auditory neurons respond maximally to a specific duration or a range of durations (frogs, Narins and Capranica, 1980; Feng et al., 1990; Gooler and Feng, 1992), (bats, Jen and Schlegel, 1982; Pinheiro et al., 1991; Casseday et al., 1994, 2000; Ehrlich et al., 1997; Galazyuk and Feng, 1997; Fuzessery and Hall, 1999; Jen and Feng, 1999; Jen and Zhou, 1999; Zhou and Jen, 2001, 2006; Faure et al., 2003; Fremouw et al., 2005; Jen and Wu, 2005, 2006, 2008; Wu and Jen, 2006, 2010), (cats, He et al., 1997), (chinchillas, Chen, 1998), (mice, Brand et al., 2000) and (rats, Perez-Gonzalez et al., 2006). The sound duration to which these neurons are tuned correspond closely to the behaviorally relevant sounds in these animal species. As such, duration-selective auditory neurons play an important role for sound recognition particularly in human speech, animal communication, and bat echolocation (Popper and Fay, 1995; Shannon et al., 1995; Covey and Casseday, 1999).

On the other hand, natural sounds such as vocal communication sounds of many animal species typically occur as temporally patterned trains of sound pulses. When stimulated with these sound pulses, the response of auditory neurons to an individual sound pulse is inevitably affected by the preceding and succeeding sounds (i.e., forward and backward masking). For example, the inferior collicular neurons show larger responses to single pulses presented in temporal isolation than to the same pulse presented in temporally patterned trains of sound pulses (Moriyama et al., 1994). Furthermore, the response size of collicular neurons progressively decreases with sequentially presented sound pulses (Pinheiro et al., 1991; Moriyama et al., 1994; Brosch and Schreiner, 1997; Lu et al., 1997, 1998; Litovsky and Yin, 1998; Jen and Zhou, 1999; Jen et al., 2001; Zhou and Jen, 2002a,b; Jen and $\mathrm{Wu}, 2005)$. These neurophysiological findings have been corroborated by behavioral studies, which show a human subject or an animal only perceives the leading source when two spatially separated clicks are presented with a brief delay within 5 ms (Wallach et al., 1949; Zurek, 1980; Freyman et al., 1991). When the delay between the two sounds is larger than $8-10 \mathrm{~ms}$ range, both the leading and lagging sounds are perceived as individual one (Freyman et al., 1991). However, longer delay of several milliseconds is required for perception of individual sounds when tested with trains of paired sounds or when each leading sound is succeeded by several echoes of various time delays
(Yost and Soderquist, 1984; Yost and Guzman, 1996). All these studies indicate that when encountered with temporally patterned trains of sequential sound pulses, the separation of individual sounds is an important temporal attribute that determines an animal's ability in perceiving individual sounds.

The neural basis underlying an animal's ability in perception of individual sounds is the recovery cycle of auditory neurons, which determines a neuron's ability in responding to closely spaced sound pulses. Many studies have shown that neurons in the inferior colliculus of various animal species have a wide range of recovery cycle (cats, Litovsky and Yin, 1998; rabbits, Fitzpatrick et al., 1995; barn owls, Keller and Takahashi, 1996; bats, Grinnell, 1963; Suga, 1964, 1970; Friend et al., 1966; Suga and Schlegel, 1973; Pollak et al., 1977; Lu et al., 1997; Zhou and Jen, 2003; Wang et al., 2008, 2010). The recovery cycle of auditory neurons is particularly important for bats, which rely on this neuronal property to determine the target distance.

During hunting, insectivorous bats emit intense ultrasonic pulses and analyse multiple parameters of the returning echoes to extract information about their prey (Griffn, 1958). They progressively shorten the duration, decrease the amplitude, lower the frequency, and increase the repetition rate of emitted pulses as they search, approach, and finally intercept insects or negotiate obstacles (Griffn, 1958; Simmons et al., 1979; Jen and Kamada, 1982; Surlykke and Moss, 2000). Because bats typically hunt together with conspecifics, a bat has to be able to differentiate the echoes of its own emitted pulses from the echoes of pulses emitted by other bats for successful prey capture. Therefore, in the real world the bat's ear is bombarded by the pulse-echo (P-E) pairs not only produced by the bat itself but also by others during hunting. Since the gap, duration, amplitude of the P-E pairs vary throughout the entire course of hunting, analysis of an echo parameter by the bat would be inevitably affected by other co-varying echo parameters produced by the bat itself and conspecifics. For example, previous studies have shown that increasing pulse repetition rate improves multiple-parametric selectivity of inferior collicular neurons (Pinheiro et al., 1991; Wu and Jen, 1996; Galazyuk et al., 2000; Jen et al., 2001; Zhou and Jen, 2002a). The increase in pulse repetition rate also elevates the minimum threshold and lengthens the response latency of most collicular and cortical neurons (Phillips et al., 1989; Chen and Jen, 1994; Jen and Chen, 1998). Furthermore, pulse duration and repetition rate profoundly influence the best delay of auditory cortical neurons of the bat in which delay tuning typically narrows and eventually disappears at higher pulse repetition rate and shorter pulse duration (Tanaka et al., 1992; Wong et al., 1992; Tanaka and Wong, 1993). For all these reasons, the important temporal attributes of sound duration and separation of individual sounds are even more obvious for bats which rely on duration selectivity for recognition of echo duration and recovery cycle for echo ranging. 
In this review article, we present our studies of duration selectivity and recovery cycle of inferior collicular neurons of the frequency-modulated (FM) bat, Eptesicus fuscus and Pipistrellus abramus, to highlight the dynamic variation of temporal signal processing of central auditory neurons. These studies use single pulses and three biologically relevant P-E pairs of combinations of three pulse durations $(1.5,4$, and $10 \mathrm{~ms})$ at varied P-E gaps that are comparable to that occurring during the search, approach and terminal phases of hunting by bats (Griffn, 1958; Simmons et al., 1979; Jen and Kamada, 1982; Surlykke and Moss, 2000). A detailed description of materials and methods can be found in these studies (Jen and Wu, 2006, 2008; Wu and Jen, 2006; Wang et al., 2008, 2010).

\section{DYNAMIC VARIATION OF ECHO DURATION SELECTIVITY OF COLLICULAR NEURONS WITH OTHER CO-VARYING PARAMETERS}

The echo duration selectivity of a collicular neuron is studied by plotting its duration tuning curves using the number of impulses in response to eight durations $(1,1.5,2,4$, $6,8,10$, and $20 \mathrm{~ms}$ ) of the echo pulses of three P-E pairs. The echo duration tuning curves of collicular neurons can be described as band-, short-, long-, and all-pass using the same criterion adopted in previous studies (Figure 1, Jen and Feng, 1999; Jen and Zhou, 1999; Wu and Jen, 2006). The collicular neurons with band-, short-, and long-pass echo duration tuning curves show a maximal number of impulses to the best duration (BD) and are, therefore, duration-selective neurons (Figures 1A-C). Neurons with all-pass echo duration tuning curves are non-duration-selective neurons because their number of impulses never varies more than $50 \%$ with sound duration (Figure 1D).

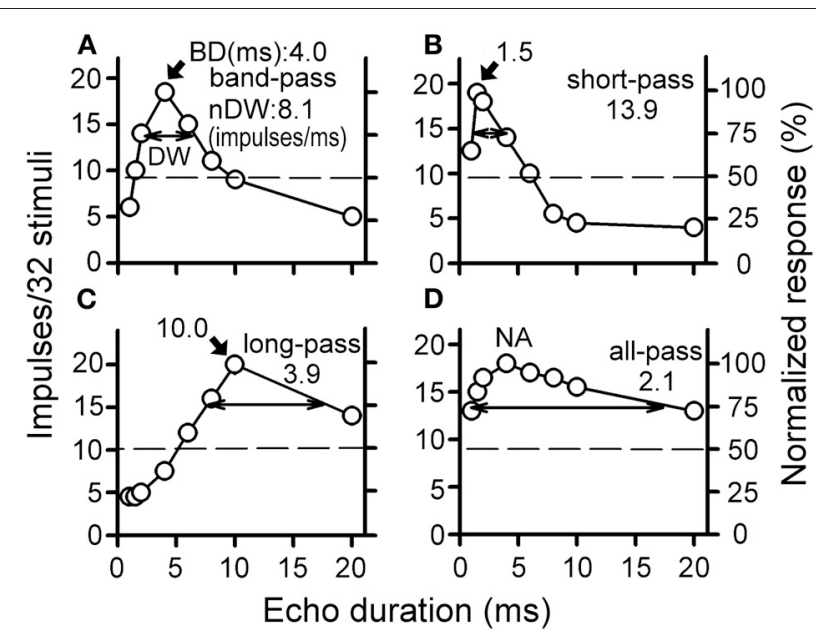

FIGURE 1 | (A) band-pass, (B) short-pass, (C) long-pass, and (D) all-pass duration tuning curves of collicular neurons of the FM bat, Eptesicus fuscus. Each horizontal dashed line indicates the 50\% maximal response. The selectivity of each echo duration curve is expressed with a best duration (BD, indicated with an arrowhead) and a normalized duration width (nDW indicated with a double-arrowheads). NA: a BD is not available [Adapted from Wu and Jen (2006)].
Because the maximal number of impulses in response to the $\mathrm{BD}$ varied greatly among individual collicular neurons, a normalized duration-width (nDW) is used to express the broadness of a duration tuning curve for consistent comparison of duration selectivity of collicular neurons obtained under different stimulation conditions. An nDW is obtained by dividing the maximum by the width of a duration tuning curve at $75 \%$ of the maximum (Figure 1, DW indicated by a double-head arrow). Thus, neurons with a large $\mathrm{nDW}$ have narrower duration tuning curve than neurons with a small nDW.

Collicular neurons have sharper duration tuning curves when determined with the echo pulses of P-E pairs than with single pulses (Figure 2). The duration tuning curves of collicular neurons becomes progressively broader with lengthening of pulse duration and P-E gap (progressive decrease in the $\mathrm{nDW}$ ). Collicular neurons have the broadest duration tuning curves when measured with single pulses (the smallest nDW). Also, a neuron's duration tuning curves may change from one type into another when measured with different P-E pairs and single pulses (Figures 2D,G,H). These observations indicate that a bat's echo duration selectivity in the real world is sharper than what is shown by earlier studies using single pulses (Jen and Schlegel, 1982; Pinheiro et al., 1991; Casseday et al., 1994, 2000; Ehrlich et al., 1997; Galazyuk and Feng, 1997; Fuzessery and Hall, 1999; Jen and Feng, 1999; Zhou and Jen, 2001).

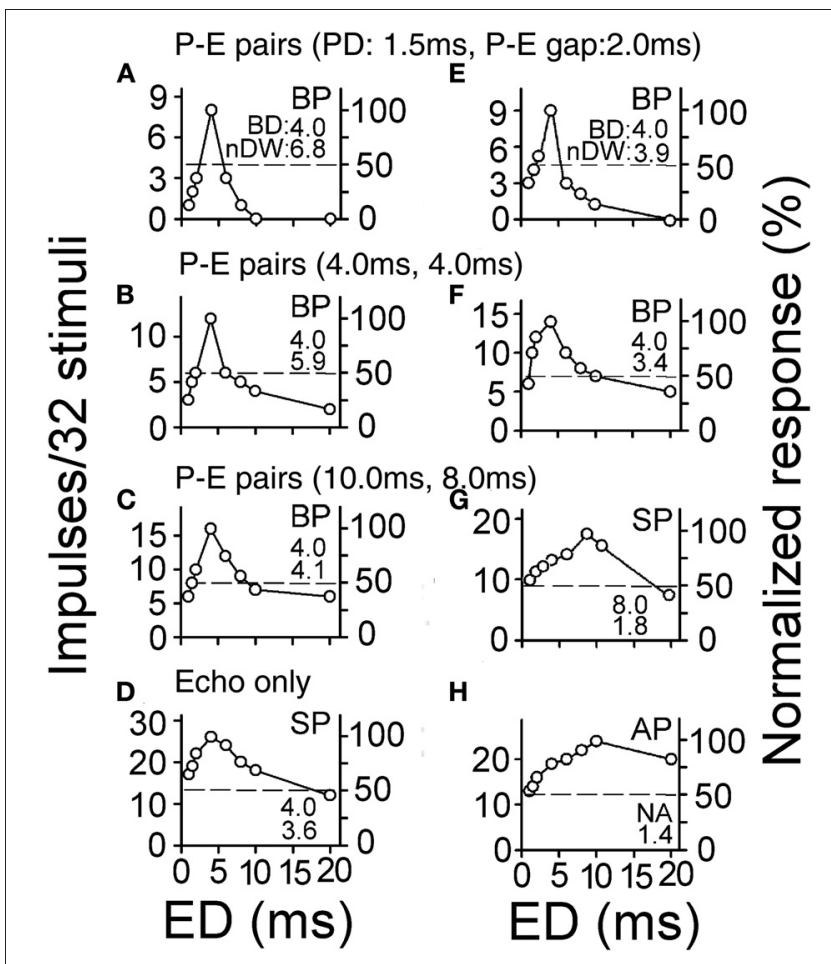

FIGURE 2 | (A-H) The echo duration tuning curves of two collicular neurons determined with the echo pulses of three pulse-echo (P-E) pairs and with single pulses. The type of duration tuning curve, the BD and the $\mathrm{nDW}$ are shown within each plot. Note that the nDW progressively decreases with lengthening of PD and P-E gap of three P-E pairs [Adapted from Wu and Jen (2006)] 
Echo duration selectivity of collicular neurons also varies with P-E amplitude difference. When studied with three biologically relevant P-E pairs at two different P-E amplitude differences, the echo duration selectivity is greater (larger $\mathrm{nDW}$ ) when determined with $4 \mathrm{~ms}$ or $10 \mathrm{~ms}$ pulse duration at $4 \mathrm{~ms}$ or $8 \mathrm{~ms} \mathrm{P}-\mathrm{E}$ gap at 20-dB than at $10-\mathrm{dB}$ amplitude difference (Figures $\mathbf{3} \mathbf{A b}, \mathbf{A c}$ vs. Bb, Bc). However, the echo duration selectivity is greater when determined with $1.5 \mathrm{~ms}$ pulse duration and $2 \mathrm{~ms}$ P-E gap at $10-\mathrm{dB}$ than at $20-\mathrm{dB}$ amplitude difference (Figures 3Bavs. Aa). These data indicate that echo duration selectivity of collicular neurons becomes sharper when echo duration progressively shortens and P-E amplitude difference decreases throughout a target approach sequence.

Collicular neurons are tonotopically organized along the dorso-ventral axis of the inferior colliculus. Their BDs are between 1.5 and $10 \mathrm{~ms}$ covering the duration of pulses emitted by the FM bat during three phases of hunting. The BD and $\mathrm{nDW}$ of collicular neurons, respectively, increases and decreases with BF and recording depth. As such, collicular neurons at upper inferior colliculus with low $\mathrm{BF}$ have short $\mathrm{BD}$ and large $\mathrm{nDW}$ while those at deeper inferior colliculus with high BF have long BD and small nDW (Jen and Wu, 2006; Wu and Jen, 2006).

\section{ECHO DURATION SELECTIVITY IS SHAPED BY GABA-MEDIATED INHIBITION}

One of the major inhibitory inputs in the inferior colliculus is mediated by GABA (Roberts and Ribak, 1987; Fubara et al., 1996). By means of application of GABA or bicuculline, which is an antagonist for $\mathrm{GABA}_{\mathrm{A}}$ receptors (Cooper et al., 1982; Bormann, 1988), many studies have shown that interaction of excitation and GABAergic inhibition contributes to auditory temporal processing and shapes multiple-parametric selectivity (e.g., duration, frequency, amplitude, direction, etc.) of collicular neurons using single repetitive sound pulses or temporally patterned trains of sound pulses (Faingold et al., 1991; Vater et al., 1992; Yang et al., 1992; Park and Pollak, 1993; Casseday et al., 1994, 2000; Klug et al., 1995; LeBeau et al., 1996; Lu et al., 1997, 1998; Koch and Grothe, 1998; Jen and Feng, 1999; Jen and Zhang, 2000; Lu and Jen, 2001; Jen et al., 2002; Zhou and Jen, 2002a,b). To study the contribution of GABAergic inhibition to echo duration selectivity of collicular neurons, echo duration tuning curves are measured before and during bicuculline and GABA application.

In agreement with previous studies (Casseday et al., 1994, 2000; Jen and Feng, 1999), bicuculline application broadens the echo duration tuning curves of collicular neurons obtained with three P-E pairs by producing a greater increase in the number of impulses for shorter and longer non-BD echo pulses than for the BD echo pulse (Figures 4A vs. B). As a result, bicuculline application changes a neuron's band-pass echo duration tuning curve into all-pass echo duration tuning curve with greatly decreased nDW (Figures 4A vs. B). Conversely, GABA application narrows the echo duration tuning curves of collicular neurons by producing a greater decrease in the number of impulses for non-BD echo pulses than for BD echo pulse (Figures $4 \mathrm{C}$ vs. D).

Although bicuculline and GABA application, respectively, broadens and narrows the duration tuning curves of collicular neurons, drug application does not change the depth-dependent duration selectivity of these neurons. The $\mathrm{nDW}$ of these neurons

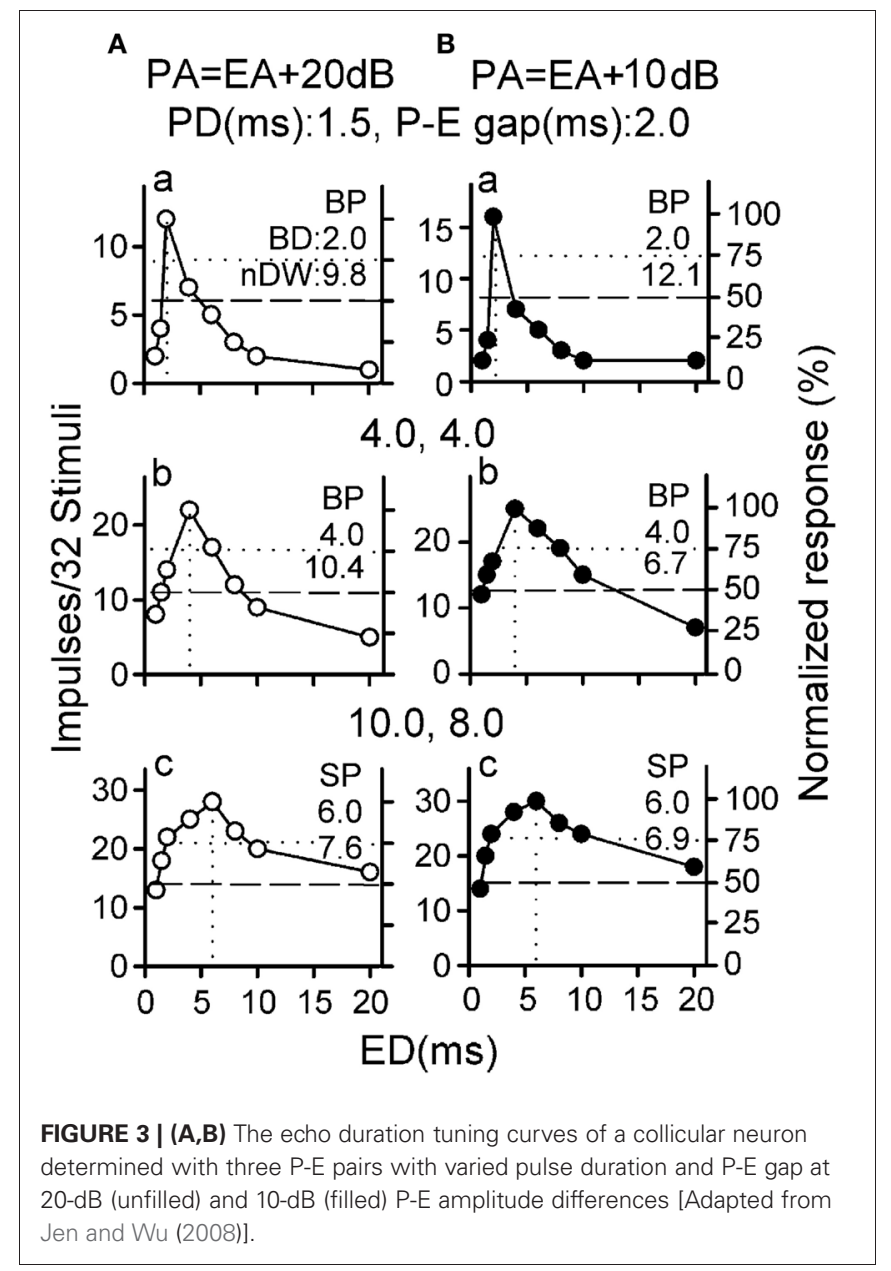

significantly decreases with recording depth both before and during drug application (Figures $\mathbf{5 A a}, \mathbf{B a}$ ). The change in $\mathrm{nDW}$ is significantly larger for neurons at upper than at deeper inferior colliculus during bicuculline application but the opposite is observed during GABA application (Figures $\mathbf{5 A b}$ vs. Bb). These observations are likely due to the fact that neurons with $\mathrm{GABA}_{\mathrm{A}}$ receptors are mostly distributed in the dorso-medial region but are sparsely distributed in the ventro-lateral region of the inferior colliculus (Fubara et al., 1996). As such, high BF neurons at deeper inferior colliculus would conceivably receive fewer GABAergic inhibitory inputs than low BF neurons at upper inferior colliculus. For this reason, bicuculline application would produce greater change and GABA application would produce lesser change in $\mathrm{nDW}$ of duration tuning curves of low $\mathrm{BF}$ neurons at upper inferior colliculus than high BF neurons at deeper inferior colliculus. In sum, these data suggest that echo duration selectivity of collicular neurons shaped by GABAergic inhibition is systematically organized along the dorso-ventral axis of the inferior colliculus.

Previous studies show that glycine-mediated inhibition also shapes the duration selectivity of collicular neurons and neurons with glycine receptors are mostly distributed at the ventro-lateral region of the inferior colliculus but are sparsely distributed at dorso-medial region (Fubara et al., 1996; Ehrlich et al., 1997). 


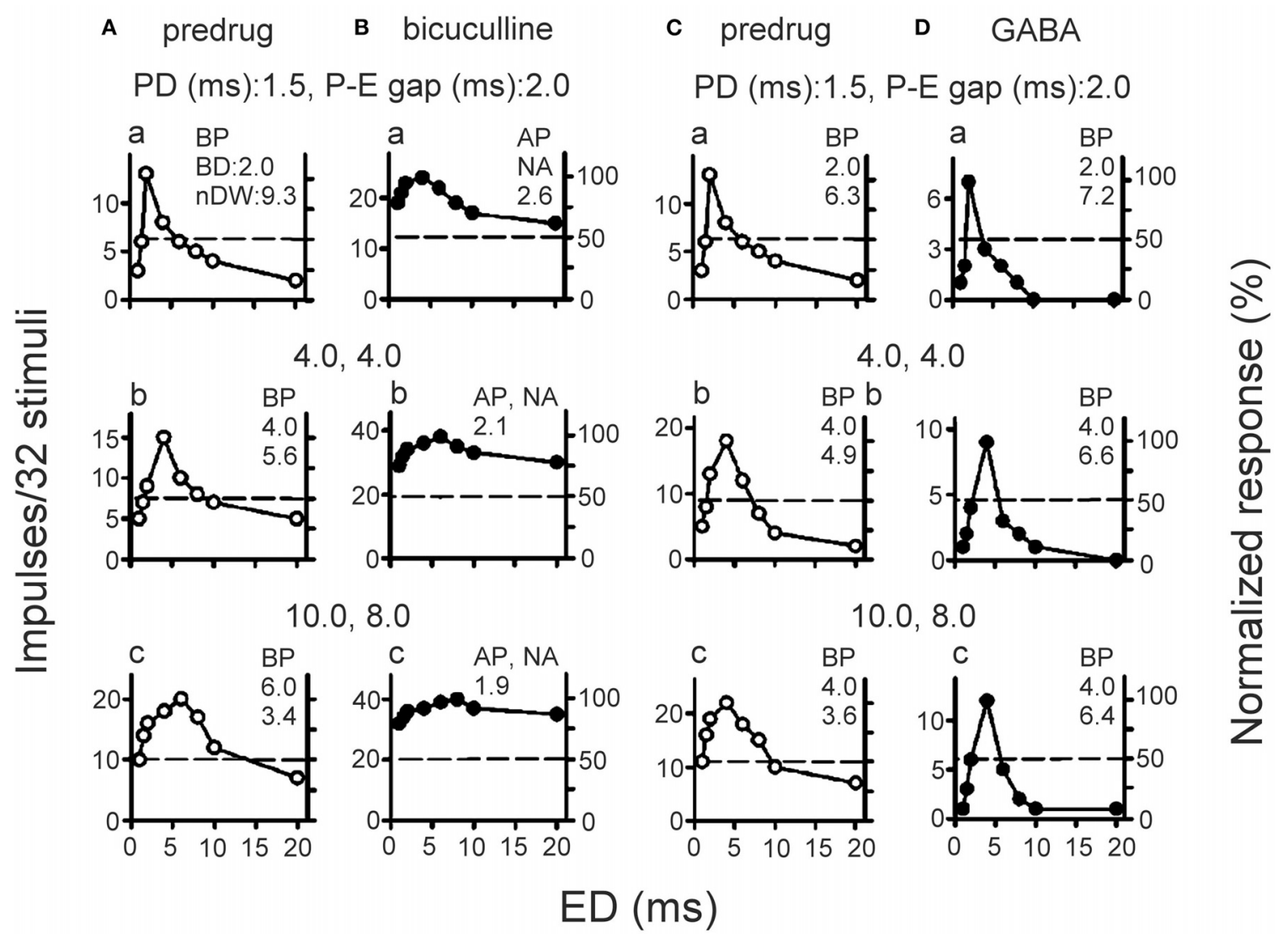

FIGURE 4 | (A,B) Echo duration tuning curves of two collicular neurons determined with three P-E pairs of varied pulse duration and P-E gap before (predrug, A,C) and during bicuculline (B) or GABA (D) application [Adapted from Wu and Jen (2006)].

Therefore, it is conceivable that glycine-mediated duration selectivity of collicular neurons may also be systematically organized along the dorso-ventral axis of the inferior colliculus. Future work is needed to determine whether GABA- and glycine-mediated duration selectivity of collicular neurons is organized systematically but oppositely along the distribution gradients of $\mathrm{GABA}_{\mathrm{A}}$ and glycine receptors.

\section{DYNAMIC VARIATION OF THE RECOVERY CYCLE OF DURATION-SELECTIVE COLLICULAR NEURONS WITH P-E AMPLITUDE DIFFERENCE}

A neuron's recovery cycle is measured by calculating the percent ratio of the neuron's number of impulses in response to the echo relative to the pulse at different $\mathrm{P}$-E intervals and amplitude difference. The bat's collicular neurons have a wide range of recovery cycle covering the P-E intervals occurring throughout the entire sequence of hunting (Suga, 1964; Friend et al., 1966; Suga and Schlegel, 1973; Pollak et al., 1977; Lu et al., 1997; Wang et al., 2008, 2010). In general, a neuron's recovery cycle increases with P-E duration and P-E amplitude difference.

To study the dynamic temporal processing of sound duration and separation of individual sounds, the dynamic variation of the recovery cycle of 30 duration-selective collicular neurons has been studied using biologically relevant P-E pairs. While the recovery cycle of 10 neurons varies unpredictably with three P-E pairs, the recovery cycle of 20 neurons varies predictably with $\mathrm{P}-\mathrm{E}$ pairs in relation to the $\mathrm{BD}$ and $\mathrm{P}-\mathrm{E}$ amplitude difference (Figures 6A,B). Specifically, neurons with 1-2 ms BD recover rapidly when stimulated with P-E pairs at $10 \mathrm{~dB}$ amplitude difference and the duration of both $\mathrm{P}$ and $\mathrm{E}$ matches the $\mathrm{BD}$ (i.e., $\mathrm{P}-\mathrm{E}$ pairs in the terminal phase, Figure 6A-2, filled arrow, solid circles). Conversely, neurons with 8-20 ms BD recover rapidly when stimulated with P-E pairs at $20 \mathrm{~dB}$ amplitude difference and the duration of both $\mathrm{P}$ and $\mathrm{E}$ matches the $\mathrm{BD}$ (i.e., P-E pairs at the search phase, Figure 6B-3, filled arrow, unfilled circles). However, neurons with 4-6 ms BD recover rapidly when stimulated with $4 \mathrm{~ms}$ of $\mathrm{P}$ and $\mathrm{E}$ at both 10 and $20 \mathrm{~dB}$ amplitude differences (i.e., $\mathrm{P}$-E pairs at the approach phase). These observations indicate that duration selectivity and recovery cycle of most collicular neurons of the bat co-vary when the echo duration and P-E amplitude difference progressively decrease throughout the entire course of hunting.

\section{POSSIBLE NEURAL MECHANISM UNDERLYING DYNAMIC TEMPORAL SIGNAL PROCESSING}

A previous study examines the interaction of excitation and inhibition in collicular neurons using a probe (excitatory pulse) and a masker (inhibitory pulse) (Lu and Jen, 2002). This study shows 


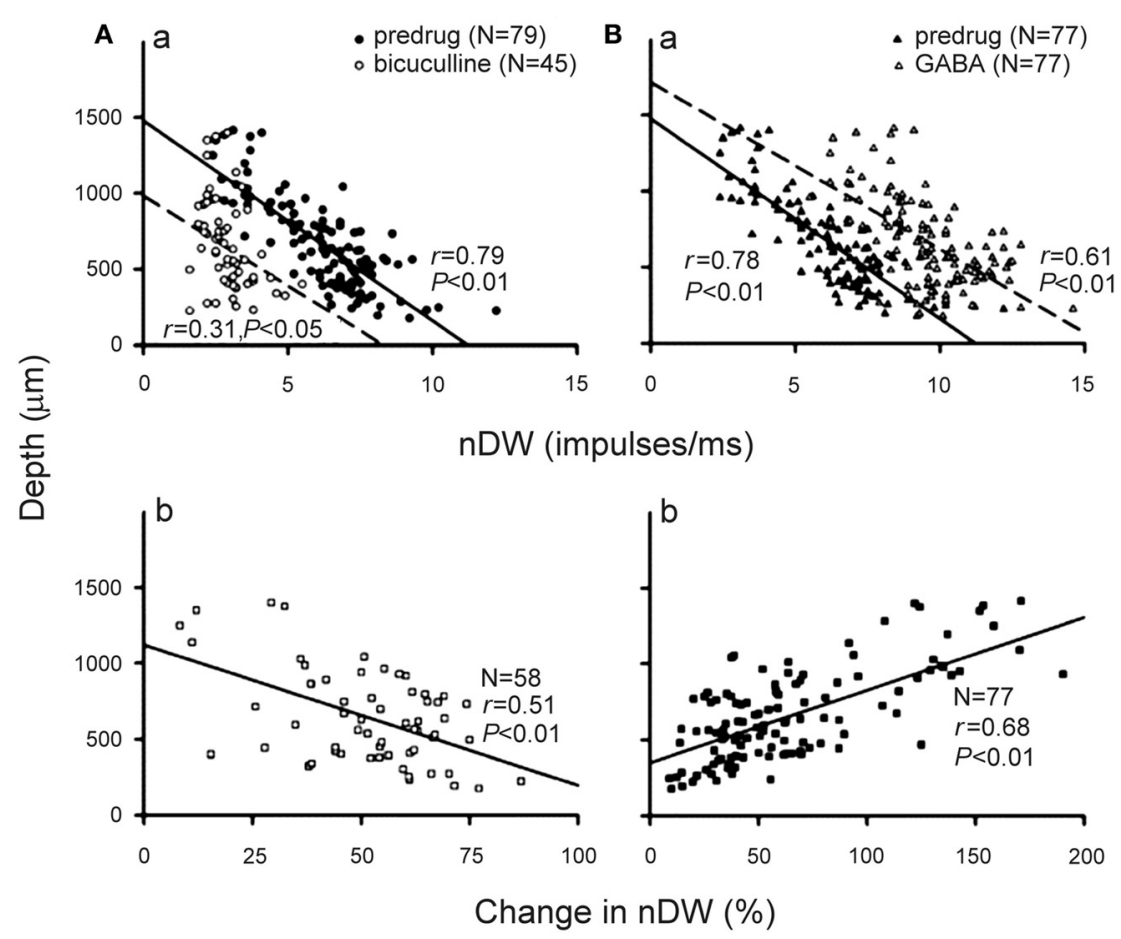

FIGURE 5 | (Aa,Ba) The distribution of nDW of collicular neurons obtained before (filled circles and triangles) and during (unfilled circles and triangles) bicuculline ( $\mathbf{A a})$ and GABA $(\mathbf{B a})$ application in relation to the recording depth.
$(\mathbf{A b}, \mathbf{B b})$ The distribution of percent change in $\mathrm{nDW}$ in relation to the recording depth. The linear regression line and correlation coefficient for each plot are shown by a solid line and $r$ [From Jen and Wu (2006)].

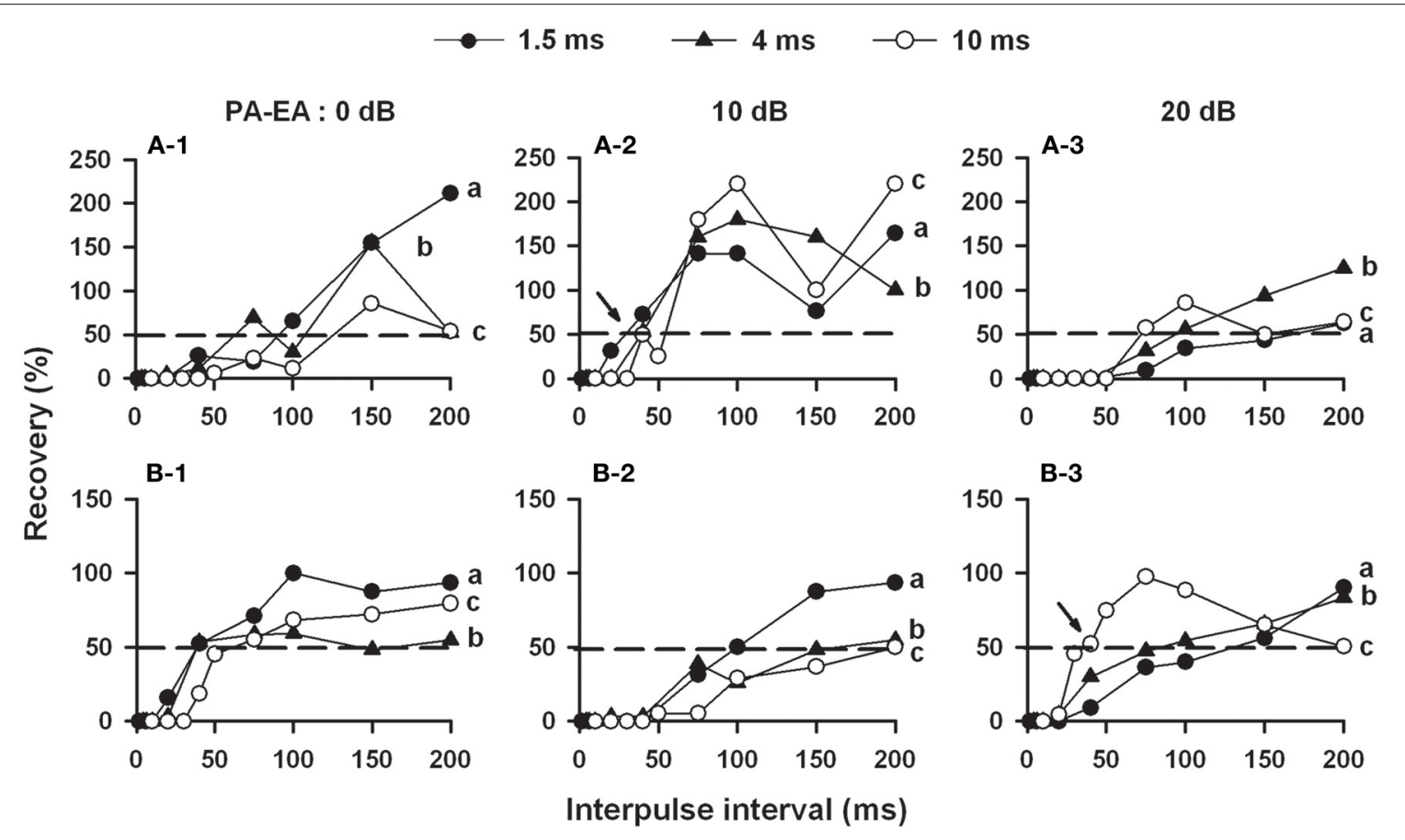

FIGURE 6 | (A) The recovery cycle of two duration-selective collicular neurons measured with P-E pairs that vary in P-E gap, duration, and amplitude differences (PA-EA $=0,10$, and $20 \mathrm{~dB}$ ). The BDs of these two neurons are $1.5 \mathrm{~ms}(\mathbf{A})$ and $10 \mathrm{~ms}(\mathbf{B})$. Note that neuron $A$ has the shortest
$50 \%$ recovery time (dashed line) when stimulated with $1.5 \mathrm{~ms}$ of $\mathrm{P}$ and $\mathrm{E}$ at 10-dB P-E amplitude difference (A-2, arrow) while neuron $B$ recovers rapidly when stimulated with $10 \mathrm{~ms}$ of $\mathrm{P}$ and $\mathrm{E}$ at 20-dB P-E amplitude difference (B-3, arrow) [Adapted from Wang et al. (2008)]. 
that masking of probe-elicited responses of collicular neurons occurs when a masker is presented within a certain inter-pulse intervals (the temporal window) in relation to the probe. Within the temporal window, the strength of this forward masking increases with shortening of inter-pulse interval. Other studies have also shown that temporal interaction of excitation and GABAergic inhibition shapes the duration selectivity and recovery cycle of collicular neurons (Casseday et al., 1994; Ehrlich et al., 1997; Lu et al., 1997; Wu and Jen, 1998, 2006; Lu and Jen, 2002; Faure et al., 2003; Zhou and Jen, 2003; Jen and Wu, 2005, 2006, 2008).

It is conceivable that when stimulated with P-E pairs, a neuron's recovery cycle is shaped by the GABA-mediated forward masking, in which the $\mathrm{P}$ serves as the masker and the $\mathrm{E}$ serves as the probe. As such, the probe-elicited response is dependent on the duration of $\mathrm{P}$ and $\mathrm{E}$, the gap and amplitude difference between $\mathrm{P}$ and $\mathrm{E}$. The forward masking and the recovery cycle may be the two predominant factors to shape a neuron's echo duration selectivity. Conceivably, when stimulated with the BD and P-E amplitude difference, integration of excitation and inhibition must result in an optimal time window within which neurons respond maximally and recover rapidly. This forward masking may also account for the sharper echo duration selectivity of collicular neurons obtained with echo pulses of P-E pairs than with single echo pulses.

\section{BIOLOGICAL RELEVANCE OF DYNAMIC TEMPORAL SIGNAL PROCESSING}

Insectivorous bats emit ultrasonic signals and analyse the returning echoes to extract information about the target range, shape, and location (Griffn, 1958). The highly successful hunting suggests that the bat's auditory system must be able to encode the changes in echo parameters produced by the bat and its conspecifics during a target approaching sequence. Whereas duration selectivity of auditory neurons underlies a bat's ability in recognition of sound duration, the recovery cycle of auditory neurons determines a neuron's ability in responding to closely spaced sound pulses. Therefore, the recovery cycle of neurons underlies the bat's ability in encoding the pulse repetition rate so as to analyse the order of individual sound pulses.

To perform effective recognition of echo duration and echo ranging, a bat must be able to encode progressive variation of

\section{REFERENCES}

Bormann, J. (1988). Electrophysiology of $\mathrm{GABA}_{\mathrm{A}}$ and $\mathrm{GABA}_{\mathrm{B}}$ receptor subtypes. Trends Neurosci. 11, $112-116$.

Brand, A., Urban, A., and Grothe, B. (2000). Duration tuning in mouse auditory midbrain. J. Neurophysiol. 84, 1790-1799.

Brosch, M., and Schreiner, C. E. (1997). Time course of forward masking tuning curves in cat primary auditory cortex. J. Neurophysiol. 77, 923-943.

Casseday, J. H., Ehrlich, D., and Covey, E. (1994). Neural tuning for sound

duration and repetition rate of emitted pulses and returning echoes throughout the entire course of hunting. Our studies show that the bat's collicular neurons have a wide range of $\mathrm{BD}$ and recovery cycle covering pulse durations and P-E intervals occurring during different phases of hunting. In addition, a neuron's duration selectivity is closely correlated with its recovery cycle such that the recovery cycle of most duration-selective neurons varies predictably with biologically relevant P-E duration, interval and amplitude difference. Conceivably, high best frequency neurons with long $\mathrm{BD}$ would be suitable for echo recognition during search and approach phases of hunting when the returning echoes are high in frequency, large in P-E amplitude difference, long in duration but low in repetition rate. Conversely, low best frequency neurons with shorter BD and sharper duration selectivity would be suitable for echo recognition during the terminal phase of hunting when the highly repetitive echoes are low in frequency, small in P-E amplitude difference and short in duration. This dynamic variation of echo duration selectivity and recovery cycle of collicular neurons may serve as the neural basis underlying successful prey capture by bats. Since these duration-selective collicular neurons are tonotopically organized, this organization would conceivably facilitate the recruitment of different groups of collicular neurons for effective processing of the returning echoes throughout a target approaching sequence.

In our studies we use insectivorous bats as the mammalian model to examine the dynamic temporal processing in the inferior colliculus. Because the layout of the auditory pathway of a bat is fundamentally similar to that of other mammals, the dynamic variation of duration selectivity and recovery cycle of collicular neurons observed in these studies conceivably also occurs in other mammalian species. Future work is necessary to test this contention.

\section{ACKNOWLEDGMENTS}

We thank two anonymous reviewers for reading an earlier version of this article. The research works described in and the preparation of this review article have been supported by the Research Board, Research Council, College of Arts and Sciences, and Division of Biological Sciences of University of MissouriColumbia, USA to Jen, the National Research Council of ROC to Wu (NSC 100-2311-B-003-002, NSC 100NPP001-1) and from the National Science Foundation of PRC to Wang (\#31000493).

auditory neurons. Brain Res. 654, 155-158.

Cooper, J. R., Bloom, F. E., and Roth R. H. (1982). The Biomedical Basis of Neuropharmacology. Oxford, New York, NY: Oxford University Press.

Covey, E., and Casseday, J. H. (1999). Timing in the auditory system of the bats. Annu. Rev. Physiol. 61, 457-476.

Ehrlich, D., Casseday, J. H., and Covey, E. (1997). Neural tuning to sound duration in the inferior colliculus of the big brown bat, Eptesicus fuscus. J. Neurophysiol. 77, 2360-2372.
Faingold, C. L., Anderson, C. A. B., and Caspary, D. M. (1991). Involvement of GABA in acoustically-evoked inhibition in inferior colliculus neurons. Hear. Res. 52, 201-216.

Faure, P. A., Fremouw, T., Casseday, J. H., and Covey, E. (2003). Temporal masking reveals properties of sound-evoked inhibition in duration-tuned neurons of the inferior colliculus. J. Neurosci. 23, 3052-3065.

Feng, A. S., Hall, J. C., and Gooler, D. M. (1990). Neural basis of sound pattern recognition in anurans. Prog. Neurobiol. 34, 313-329. 
Fitzpatrick, D. C., Kuwada, S., Batra, R., and Trahiotis, C. (1995). Neural responses to simple, simulated echoes in the auditory brainstem of the unanesthetized rabbit. J. Neurophysiol. 74, 2469-2486.

Fremouw, T., Faure, P. A., Casseday, J. H., and Covey, E. (2005). Duration selectivity of neurons in the inferior colliculus of the big brown bat: tolerance to changes in sound level. J. Neurophysiol. 94, 1869-1878.

Freyman, R. L., Clifton, R. K., and Litovsky, R. Y. (1991). Dynamic processes in the precedence effect. J. Acoust. Soc. Am. 90, 874-884.

Friend, J. H., Suga, N., and Suthers, R. A. (1966). Neural responses in the inferior colliculus of echolocating bats to artificial orientation sounds and echoes. J. Cell. Physiol. 67, 319-332.

Fubara, B. M., Casseday, J. H., Covey, E., and Schwartz-Bloom, R. D. (1996). Distribution of $\mathrm{GABA}_{A}$, $\mathrm{GABA}_{\mathrm{B}}$, and glycine receptors in the central auditory system of the big brown bat, Eptesicus fuscus. J. Comp. Neurol. 369, 83-92.

Fuzessery, Z. M., and Hall, J. C. (1999). Sound duration selectivity in the pallid bat inferior colliculus. Hear. Res. 137, 137-154.

Galazyuk, A. V., and Feng, A. S. (1997). Encoding of sound duration by neurons in the auditory cortex of the little brown bat, Myotis lucifugus. J. Comp. Physiol. A 180, 301-311.

Galazyuk, A. V., Liano, D., and Feng, A. S. (2000). Temporal dynamics of acoustic stimuli enhance amplitude tuning of inferior colliculus neurons. J. Neurophysiol. 83, 128-138.

Gooler, D. M., and Feng, A. S. (1992). Temporal coding in frog auditory midbrain: the influence of duration and rise-fall time on processing of complex amplitude-modulated stimuli. J. Neurophysiol. 67, 1-22.

Griffn, D. R. (1958). Listening in the Dark. New Haven, CT: Yale University Press. (Reprinted by Dover Publications, New York, NY, 1974).

Grinnell, A. D. (1963). The neurophysiology of audition in bats: temporal parameters. J. Physiol. 167, 67-96.

He, J. F., Hashikawa, T., Ojima, H., and Kinouchi, Y. (1997). Temporal integration and duration tuning in the dorsal zone of cat auditory cortex. J. Neurosci. 17, 2615-2625.

Jen, P. H. S., and Chen, Q. C. (1998). The effect of pulse repetition rate, pulse intensity, and bicuculline on the minimum threshold and latency of bat inferior collicular neurons. J. Comp. Physiol. A 182, 455-465.
Jen, P. H. S., and Feng, R. B. (1999). Bicuculline application affects discharge pattern and pulse-duration tuning characteristics of bat inferior collicular neurons. J. Comp. Physiol. A 184, 185-194.

Jen, P. H. S., and Kamada, T. (1982) Analysis of orientation signals emitted by the CF-FM bat, Pteronotus parnellii parnellii and the FM bat, Eptesicus fuscus during avoidance of moving and stationary obstacles. J. Comp. Physiol. A 148, 389-398.

Jen, P. H. S., and Schlegel, P. (1982). Auditory physiological properties of the neurons in the inferior colliculus of the big brown bat, Eptesicus fuscus. J. Comp. Physiol. A 147, 351-363.

Jen, P. H. S., and Wu, C. H. (2005). The role of GABAergic inhibition in shaping the response size and duration selectivity of bat inferior collicular neurons to sound pulses in rapid sequences. Hear. Res. 202, 222-234.

Jen, P. H. S., and Wu, C. H. (2006). Duration selectivity organization in the inferior colliculus of the big brown bat, Eptesicus fuscus. Brain Res. 1108, 76-87.

Jen, P. H. S., and Wu, C. H. (2008). Echo duration selectivity of the bat varies with pulse-echo amplitude difference. Neuroreport 19, 373-377.

Jen, P. H. S., and Zhang, J. P. (2000). The role of GABAergic inhibition on direction dependent sharpening of frequency tuning in bat inferior collicular neurons. Brain Res. 862, 127-137.

Jen, P. H. S., and Zhou, X. M. (1999). Temporally patterned sound trains affect duration tuning characteristics of bat inferior collicular neurons. J. Comp. Physiol. A 185, 471-478.

Jen, P. H. S., Wu, F. J., and Chen, Q. C. (2002). The effect of two-tone stimulation on responses of two simultaneously recorded neurons in the inferior colliculus of the big brown bat, Eptesicus fuscus. Hear. Res. 168, 139-149.

Jen, P. H. S., Zhou, X. M., and Wu, C. H. (2001). Temporally patterned pulse trains affect frequency tuning and intensity coding of inferior collicular neurons of the big brown bat, Eptesicus fuscus. J. Comp. Physiol. A 187, 605-616.

Jen, P. H.-S., Wu, C. H., Luan, R. H., and Zhou, X. M. (2002). GABAergic inhibition contributes to pulse repetition rate-dependent frequency selectivity in inferior colliculus of the big brown bat, Eptesicus fuscus. Brain Res. 948, 159-164.
Keller, C. H., and Takahashi, T. T. (1996). Response to simulated echoes by neurons in the barn owl's auditory space map. J. Comp. Physiol. A 178, 499-512.

Klug, A., Park, T. J., and Pollak, G. D. (1995). Glycine and GABA influence binaural processing in the inferior colliculus of the mustache bat. J. Neurophysiol. 74, 1701-1713.

Koch, U., and Grothe, B. (1998). GABAergic and glycinergic inhibition sharpens tuning for frequency modulations in the inferior colliculus of the big brown bat. J. Neurophysiol. 80, 71-82.

LeBeau, F. E., Malmierca, M. S., and Rees, A. (2001). Iontophoresis in vivo demonstrates a key role for GABAA and glycinergic inhibition in shaping frequency response areas in the inferior colliculus of guinea pig. J. Neurosci. 21, 7303-7312.

LeBeau, F. E., Rees, A., and Malmierca, M. S. (1996). Contribution of GABA- and glycine-mediated inhibition to the monaural temporal response properties of neurons in the inferior colliculus. J. Neurophysiol. 75, 902-919.

Litovsky, R. Y., and Yin, T. C. (1998). Physiological studies of the precedence effect in the inferior colliculus of the cat. I. Correlates of psychophysics. J. Neurophysiol. 80, 1285-1301.

Lu, Y., Jen, P. H. S., and Wu, M. (1998). GABAergic disinhibition affects the responses of bat inferior collicular neurons to temporally patterned sound pulses. J. Neurophysiol. 79, 2303-2315.

Lu, Y., Jen, P. H. S., and Zheng, Q. Y. (1997). GABAergic disinhibition changes the recovery cycle of bat inferior colliculus neurons. J. Comp. Physiol. A 181, 331-341.

Lu, Y., and Jen, P. H. S. (2001). GABAergic and glycinergic neura inhibition in excitatory frequency tuning of bat inferior collicular neurons. Exp. Brain Res. 14, 331-339.

Lu, Y., and Jen, P. H. S. (2002) Interaction of excitation and inhibition in inferior collicular neurons of the big brown bat, Eptesicus fuscus. Hear. Res. 169, 140-150.

Moriyama, T., Hou, T.-Z., Wu, M., and Jen, P. H. S. (1994). Responses of inferior collicular neurons of the FM bat, Eptesicus fuscus to pulse trains with varied pulse amplitudes. Hear. Res. 79, 105-114.

Narins, P. M., and Capranica, R. R. (1980). Neural adaptation for processing the two-note call of the Puerto Rican treefrog, Eleutherodactylus coqui. Brain Behav. Evol. 17, 48-66.
Park, T. J., and Pollak, G. D. (1993). GABA shapes sensitivity to interaural intensity disparities in the mustache bat's inferior colliculus: implications for encoding sound location. J. Neurosci. 13, 2050-2067.

Perez-Gonzalez, D., Malmierca, M. S., Moore, J. M., Hernandez, O., and Covey, E. (2006). Duration selective neurons in the inferior colliculus of the rat: topographic distribution and relation of duration sensitivity to other response properties. J. Neurophysiol. 95, 823-836.

Phillips, D. P., Hall, S. E., and Hollett, J. L. (1989). Pulse repetition rate and signal level effects on neuronal responses to brief pulses in cat auditory cortex. J. Acoust. Soc. Am. 85, 2537-2549.

Pinheiro, A. D., Wu, M., and Jen, P. H. S. (1991). Encoding repetition rate and duration in the inferior colliculus of the big brown bat, Eptesicus fuscus. J. Comp. Physiol. A 169, 69-85.

Pollak, G. D., Bodenhamer, R., Marsh, D. S., and Souther, A. (1977). Recovery cycles of single neurons in the inferior colliculus of unanesthetized bats obtained with frequency-modulated and constantfrequency sounds. J. Comp. Physiol. A 120, 215-250.

Popper, A. N., and Fay, R. R. (eds). (1995). Hearing by Bats. Berlin, Heidelberg, New York: Springer.

Roberts, R. C., and Ribak, C. E. (1987). An electron microscopic study of GABAergic neurons and terminals in the central nucleus of the inferior colliculus of the cat. J. Neurocytol. $16,333-345$.

Shannon, R. V., Zeng, F. G., Kamath, V., Wygonski, J., and Ekelid, M. (1995). Speech recognition with primary temporal cues. Science 270 303-304.

Simmons, J. A., Fenton, M. B. and O'Farrell, M. J. (1979). Echolocation and pursuit of prey by bats. Science 203, 16-21.

Suga, N. (1964). Recovery cycles and responses to frequency modulated tone pulses in auditory neurons of echolocating bats. J. Physiol. 175, 50-80.

Suga, N. (1970). Echo-ranging neurons in the inferior colliculus of bats. Science 170, 449-452.

Suga, N., and Schlegel, P. (1973). Coding and processing in the auditory system of FM signal producing bats. J. Acoust. Soc. Am. 54, 174-190.

Surlykke, A., and Moss, C. F. (2000). Echolocation behavior of big brown bat, Eptesicus fuscus, in the field and 
the laboratory. J. Acoust. Soc. Am. 108, 2419-2429.

Tanaka, H., Wong, D., and Taniguchi, I. (1992). The influence of stimulus duration on the delay tuning of crotical neurons in the FM bat, Myotis lucifugus. J. Comp. Physiol. A 171, 29-40.

Tanaka, H., and Wong, D. (1993). The influence of temporal pattern of stimulation on delay tuning of neurons in the auditory cortex of the FM bat, Myotis lucifugus. Hear. Res. 66, 58-66.

Vater, M., Habbicht, H., Kossl, M., and Grothe, B. (1992). The functional role of GABA and glycine in monaural and binaural processing in the inferior colliculus of horseshoe bats. J. Comp. Physiol. A 171, 541-553.

Wallach, H., Newman, E. B., and Rosenzweig, M. R. (1949). The precedence effect in sound localization. J. Am. Psychol. 57, 315-336.

Wang, X., Luo, F., Wu, F. J., Chen, Q. C., and Jen, P. H. S. (2008). The recovery cycle of bat durationselective collicular neurons varies with hunting phase. Neuroreport 19, 861-865.

Wang, X., Luo, F., Wu, F. J., Jen, P. H. S., and Chen, Q. C. (2010). The recovery cycle of neurons in the inferior colliculus of the FM bat determined with varied pulse-echo duration and amplitude. Chin. J. Physiol. 53, 119-129.

Wong, D., Maekawa, M., and Tanaka H. (1992). The effect of pulse repetition rate on the delay sensitivity of neurons in the auditory cortex of the FM bat, Myotis lucifugus. J. Comp. Physiol. A 170, 393-402.

Wu, C. H., and Jen, P. H. S. (2006) GABA-mediated echo duration selectivity of inferior collicular neurons of Eptesicus fuscus, determined with single pulses and pulse-echo pairs. J. Comp. Physiol. A 192, 985-1002.

Wu, C. H., and Jen, P. H. S. (2010). A duration coding mechanism underlying bat echo recognition. Adapt. Med. 2, 71-77.

Wu, M., and Jen, P. H. S. (1996). Temporally patterned sound pulses affect directional sensitivity of inferior collicular neurons of the big brown bat, Eptesicus fuscus. J. Comp. Physiol. A 179, 385-393.

Wu, M., and Jen, P. H.-S. (1998). The recovery properties of neurons in the inferior colliculus, auditory cortex and the pontine nuclei of the big brown bat, Eptesicus fuscus. Chin. J. Physiol. 41, 1-8.

Yang, L., Pollak, G. D., and Resler, C. (1992). GABAergic circuits sharpen tuning curves and modify response properties in the mustache bat inferior colliculus. J. Neurophysiol. 68 1760-1774.

Yost, W. A., and Guzman, S. J. (1996). Auditory processing of sound sources: is there an echo in here? Curr. Dir. Psychol. Sci. 5, 125-131.

Yost, W. A., and Soderquist, D. R. (1984). The precedence effect: revisited. J. Acoust. Soc. Am. 76, 1377-1383.

Zhou, X. M., and Jen, P. H. S. (2001). The effect of sound intensity on duration-tuning characteristics on bat inferior collicular neurons. J. Comp. Physiol. A 187, 63-73.

Zhou, X. M., and Jen, P. H. S. (2002a) The role of GABAergic inhibition in shaping directional selectivity of bat inferior collicular neurons determined with temporally patterned pulse trains. J. Comp. Physiol. A 188 815-826.

Zhou, X. M., and Jen, P. H. S. (2002b) The effect of sound duration on rate-intensity functions of inferior collicular neurons of the big brown bat, Eptesicus fuscus. Hear. Res. 166, 124-135.

Zhou, X. M., and Jen, P. H. S. (2003). The effect of bicuculline application on azimuth dependent recovery cycle of inferior collicular neurons of the big brown bat,
Eptesicus fuscus. Brain Res. 973, 131-141.

Zhou, X. M., and Jen, P. H. S. (2006). Duration selectivity of bat inferior collicular neurons improves with increasing repetition rate. Chin. J. Physiol. 49, 46-55.

Zurek, P. M. (1980). The precedence effect and its possible role in the avoidance of interaural ambiguities. J. Acoust. Soc. Am. 67, 952-964.

Conflict of Interest Statement: The authors declare that the research was conducted in the absence of any commercial or financial relationships that could be construed as a potential conflict of interest.

Received: 25 February 2012; accepted: 20 April 2012; published online: 08 May 2012.

Citation: Jen PH-S, Wu CH and Wang X (2012) Dynamic temporal signal processing in the inferior colliculus of echolocat ing bats. Front. Neural Circuits 6:27. doi: 10.3389/fncir.2012.00027

Copyright (C) 2012 Jen, Wu and Wang. This is an open-access article distributed under the terms of the Creative Commons Attribution Non Commercial License, which permits non-commercial use, distribution, and reproduction in other forums, provided the original authors and source are credited. 